\title{
Daidzin-rich Soy Isoflavone Extracts Promote Estrous Cycling in VCD- induced Menopause Mouse Model
}

\author{
Simbarashe Mazambani, Kailee Johnson, Sravan Vemuri, Sameerh Alshafi, and Venugopalan Cheriyath* \\ Department of Biological and Environmental Sciences, Texas A\&M University-Commerce, USA
}

Submission: November 25, 2017; Published: January 19, 2018

*Corresponding author: Venugopalan Cheriyath, Department of Biological and Environmental Sciences Texas A\&M University-Commerce Commerce, Texas-75429, Tel: 903-468-6064; Fax: 903-886-5997; Email: venu.cheriyath@tamuc.edu

\begin{abstract}
By 2025 postmenopausal women will make up to $20 \%$ of the US population. Since traditional hormone replacement therapies (HRT) increase the risk of breast cancer, alternative strategies are needed to delay and manage menopause. With this intention, recent studies in our laboratory screened isoflavone (phytoestrogens) extracts of fifty-four commercially grown soybean cultivars in a bioassay and identified extracts from three cultivars with high daidzin to genistin ratio as highly estrogenic. Based these results, we hypothesized that soy isoflavone extracts with a high daidzin to genistin ratio will promote estrous cycling in menopausal mice. In a VCD-induced menopause mouse model, the number of estrous cycles was significantly increased in mice receiving a soy isoflavone supplemented diet compared to VCD-treated mice receiving an unfortified diet $(\mathrm{p}=0.0004)$. Moreover, soy isoflavone extracts that promoted estrous cycling had a high daidzin to genistin ratio. There was no significant difference in percentage weight change between the control mice receiving unfortified diets and mice receiving a soy isoflavone fortified diet $(\mathrm{p}=0.2)$. In addition there were no palpable mammary tumors detected in mice. Taken together, our study suggests that the composition of soy isoflavone extracts determines their effectiveness in delaying menopause. Soy isoflavone extracts with a high daidzin to genistin ratio may delay the onset of menopause by increasing functional estrogen activity during menopause transition.
\end{abstract}

Keywords: Soy isoflavones; Daidzin; Menopause; VCD-Induced menopause mouse model; Phytoestrogens; Hormone replacement therapy Abbreviations: VCD: 4-Vinylcyclohexene Diepoxide; HRT: Hormone Replacement Therapy; OVX: Ovariectomy; SIE: Soy Isoflavone Extract

\section{Introduction}

Menopause is characterized by, and defined as a cessation of cyclic menstruation [1]. Exhaustion of ovarian follicular reserves appears to drive menopause and various replenishment therapies have been advocated to supplement hormones during menopause [2,3]. As estrogen levels drop, the estrogen/androgen ratio changes in postmenopausal women, which may result in many age related diseases such as decreasing bone density, cardiovascular diseases, cognitive diseases, metabolic syndromes and possibly some cancers $[4,5]$. In the United States 4,000 women enter menopause each day and by 2025 postmenopausal women will make up to $20 \%$ of the US population [1]. When menopause symptoms become severe they can drastically reduce the quality of life of menopausal women and interfere with daily activities [6]. Hormone replacement therapy (HRT) is effective in most cases to alleviate menopause symptoms; however this option has fallen out of favor because of its association with the increased risk of developing breast cancer. Therefore, to delay menopause, phytoestrogens such as soy isoflavones have been suggested as an alternative for HRT that may also improve the quality of life of postmenopausal women [7,8]

Soy isoflavones and endogenous estrogen are similar in structure and bind to the same receptors, but the potency of soy isoflavones is estimated to be $\sim 1000$-fold lower than that of $17-\alpha$ estradiol $[7,8]$. Apart from the antioxidant effects, the estrogenic effects of soyisoflavones have been suggested to benefit the cardiovascular system, bone, and vasomotor systems in pre- and postmenopausal women [9]. Soybeans contain six types of isoflavones: three of each are glyconic (genistin, daidzin and glycitin) and aglyconic (genistein, glycitein and daidzein) [10]. Each of these isoflavones can exist in four isomeric forms with varying estrogenic activities $[7,10]$. Recently we screened soy isoflavone extracts from 54 cultivars and identified three cultivars each with high and low estrogenic activity [10].

Among isoflavones, there was a positive correlation between daidzin concentration and estrogenic activity whereas high concentration of genistin was negatively 
associated with estrogenic activity [10]. Based on these results we hypothesized that a soybean extract with a high daidzin to genistin ratio will delay the onset of menopause in mice. This hypothesis was tested in a 4 vinylcyclohexene diepoxide
(VCD)-induced menopause mouse model that closely simulates human menopause [3]. Unlike ovariectomy (OVX), menopause induced by VCD creates a well characterized model for the gradual onset of menopause $[11,12]$.

\section{Conclusion}

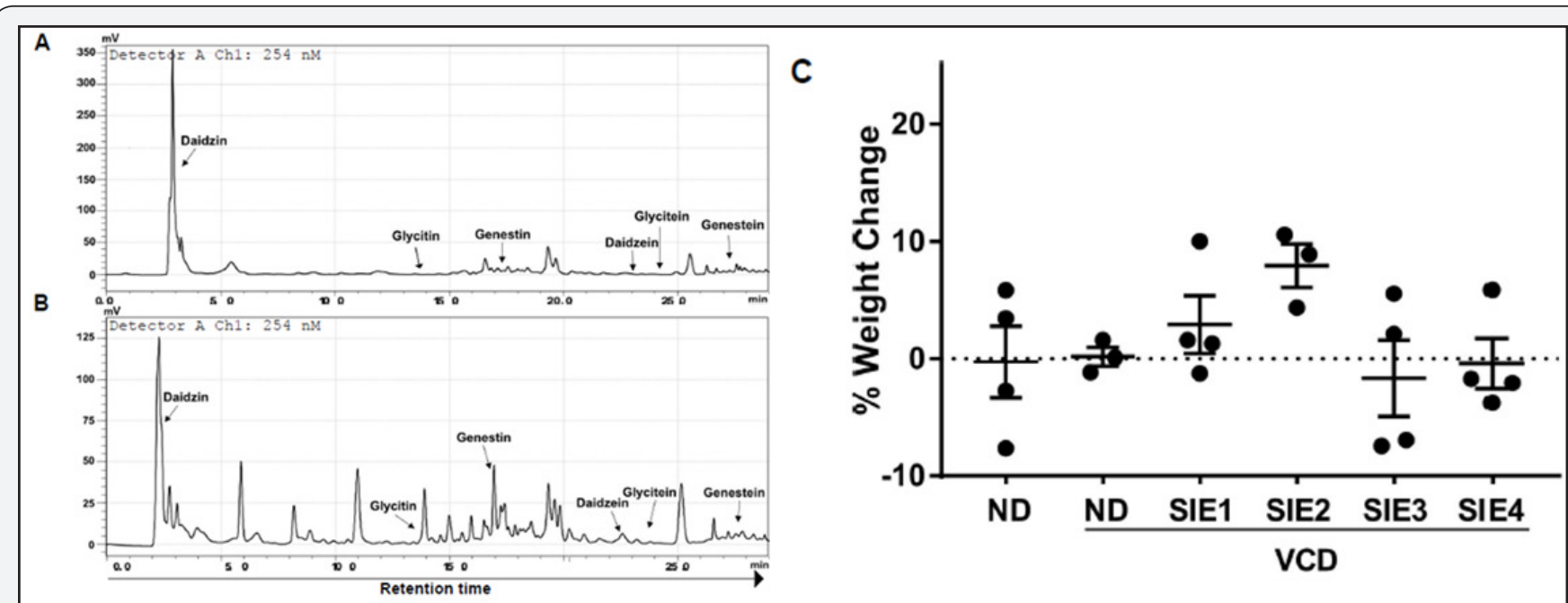

Figure 1: Effects of soy isoflavone extracts weight change in mice. A and B) Representative HPLC chromatograms of soy isoflavone extracts from soybean cultivars. Peaks were identified using standard control mixtures of isoflavones as described [10]. Chromatograms depict a cultivar with a high daidzin to genestin concentration ratio, and another with low daidzin to genestin ratio. C) Isoflavone extracts had no siginificant effect on weight change. Mice were weighed daily for the duration of the study (95 days) and percentage weight change for each mouse was calculated. Each bar on the graph represents mean \pm SEM of percentage weight for each group. A $p$ value of 0.05 is considered significant in one way ANOVA.

The overall objective of this study was to investigate the effect of soy isoflavones during transition into menopause in a VCD-induced menopause mouse model that closely mimics human menopause. Menopause was induced in seven month old mice as described $[3,13,14]$. Isoflavones were extracted from soy bean cultivars with high and low daidzin to genistin ratios that were identified in our prior study [10] and were used to fortify mouse stock feed that was devoid of any isoflavones or soy product (Harlan Teklad Global Diets 2014 $14 \%$ Protein Rodent Diet). HPLC analyses of soy isoflavone extracts identified daidzin, genestin and glycitin as the main constituents. Representative chromatograms depict a cultivar with a high daidzin to genestin concentration ratio, and another with low daidzin to genestin ratio (Figure 1A \& 1B). After obtaining IACUC approval (AUP\# P13-05-01), 24 mice were selected by randomized block design and allocated evenly into six different diet groups (Groups 1-6). Group 1 was fed a normal isoflavone free diet (ND) and received no VCD injection. Group 2-6 all received VCD injections; however group 2 was fed a normal isoflavone free diet (ND + VCD). Groups 3-6 received an isoflavone fortified diet with soy isoflavone extracts (SIE) from four different soybean cultivars (SIE1-4+ VCD). At the beginning of the investigation, a pilot study was conducted to rule out potential toxicity or any bias towards fortified diets. The average consumption of unfortified (ND) and soy isoflavone fortified diets were $109.13 \pm 2.6 \mathrm{~g}$ during the six days $(\mathrm{p}=0.42$, data not shown). Consequently, during the study period, mice in all cohorts had similar weight gain/ loss (Figure 1C).

Next, the stages of estrous cycles (proestrus, estrus, metestrus, and diestrus) were determined using a simple noninvasive method as described [15]. Proestrus phase consisted of round nucleated epithelial cells; during the estrus phase the majority of cells were cornified epithelial cells; cells in metestrus were a combination of leukocytes (arrows) and a few round cells; cells in the diestrus phase were predominantly leukocytes which contained bi lobed darkly stained nuclei (Figure 2A). The number of estrous cycles for each mouse was plotted, counted and represented (Figure 2B). One way ANOVA analyses indicated a significant difference in estrous cycles $(p=0.0002)$ among the six groups (Figure 2C). Compared to the ND group ( $8.0 \pm 0.7$ cycles) the VCD group ( $4 \pm 0$ cycles) displayed significantly decreased estrous cycling $(p<0.05)$ (Figure 2B). However cycling did not abruptly cease due to VCD, suggesting progressive onset of menopause which is consistent with prior studies $[13,16]$. Compared to the ND+VCD group, fortification of feed with a high daidzin to genistin ratio extract (SIE1+VCD and SIE2+VCD) increased estrous cycles to $11 \pm 1.1$ cycles and $8.25 \pm 0.9$ cycles respectively ( $p=0.0004$ and $\mathrm{p}=0.034$ respectively). The increased estrous cycling observed in the SIE1+VCD and SIE2+VCD groups of mice can be attributed to the estrogenic effect of the daidzin-rich SIE1 and SIE2 
extracts (Figure 2C). Estrous cycling in SIE1+VCD group was also significantly increased compared to SIE3+VCD $(4.8 \pm 0.5$ cycles) and SIE $4+V C D(5.5 \pm 1.1$ cycles) groups ( $p=0.0006$ and $\mathrm{p}=0.0052$ respectively, Figure $2 \mathrm{C}$ ). Interestingly, cohorts fed with low estrogenic isoflavone extract (SIE 3 and SIE 4) did not increase estrous cycling compared to either of the normal diet groups. SIE3 and SIE4 both contained a low daidzin to genestin ratio compared to SIE1 suggesting that the ratio of daidzin and genestin are key in determining the estrogenic effect of soy isoflavone extracts.

These results suggest that exposure to lower dosages of estrogenic compounds during premenopause may not be effective in alleviating menopause symptoms. Moreover, some isoflavones have been implicated as competitive inhibitors of estrogen during premenopause by exhibiting an antiestrogenic effect [9]. If low estrogenic isoflavones do participate in competitive inhibition during premenopause they could augment menopause symptoms instead of preventing them, therefore women approaching menopause could increase their health risks by taking soy isoflavone supplements with low estrogenic activity. The disparity of estrogenic activity among soybean cultivars, as indicated in this study, emphasizes the importance of understanding the isoflavone composition in soy products before they are advocated as supplements to manage pre- and post-menopause.

In summary, our results identified that soy isoflavone extracts with high daidzin to genistin ratio from two cultivars significantly increased estrous cycling in mice to delay VCDinduced menopause. This result suggests that these extracts may have increased functional estrogen activity during transition into menopause without significantly affecting weight changes in mice and without producing any evident palpable mammary tumors. In addition our results suggest that some soy isoflavone extracts with low estrogenic activity and low daidzin to genistin ratio may not be effective for alleviating menopause symptoms and as potential alternatives for HRT in menopausal women (Figure $1 \& 2$ ).

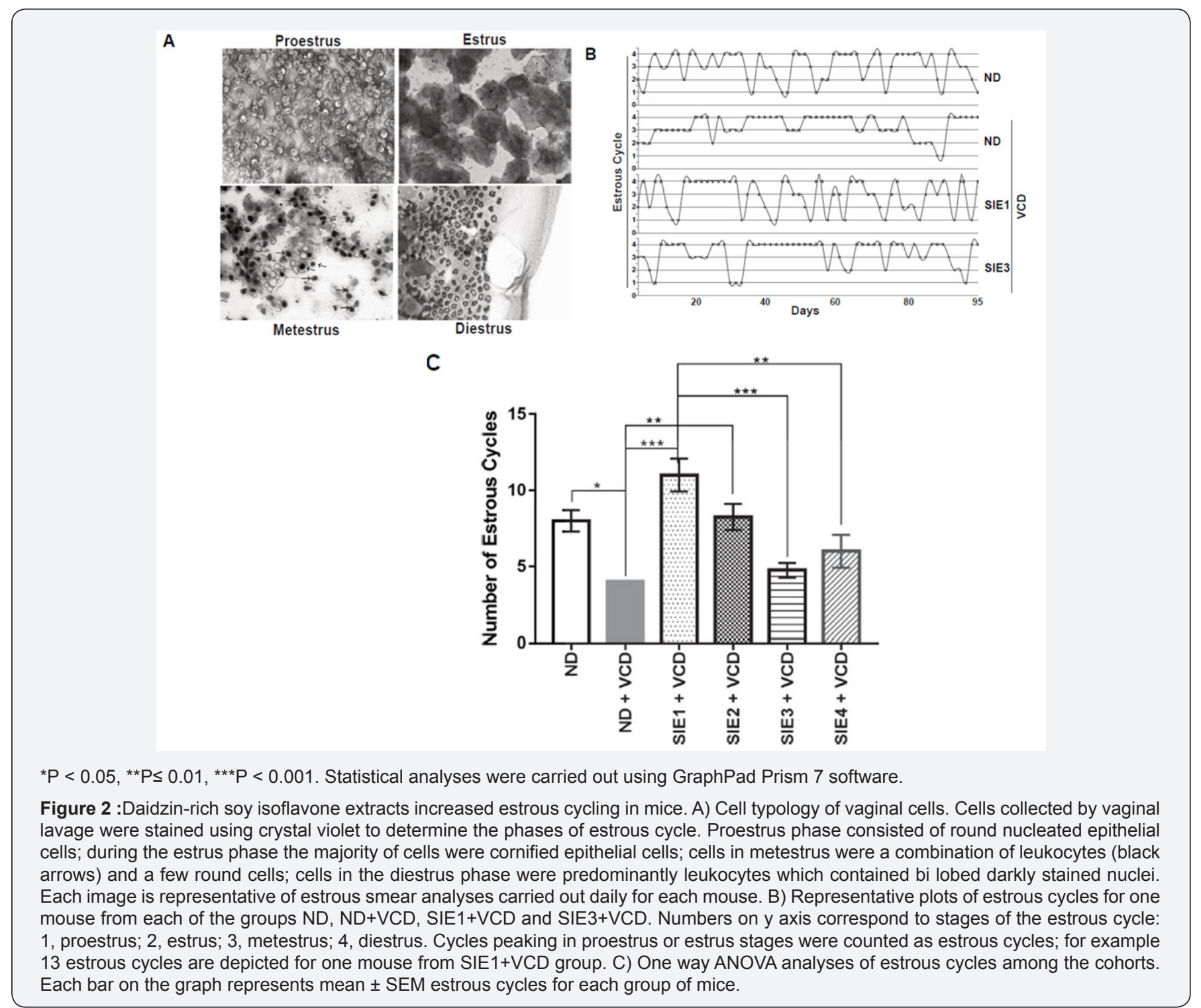




\section{References}

1. Wu JM, Zelinski MB, Ingram DK, Ottinger MA (2005) Ovarian Aging and Menopause: Current Theories, Hypotheses, and Research models. Exp Biol Med (Maywood) 230(11): 818-828.

2. Patisaul HB, Jefferson W (2010) The pros and cons of phytoestrogens. Front Neuroendocrinol 31(4): 400-419.

3. Romero-Aleshire MJ, Diamond-Stanic MK, Hasty AH, Hoyer PB Brooks HL (2009) Loss of ovarian function in the VCD mouse-model of menopause leads to insulin resistance and a rapid progression into the metabolic syndrome. Am J Physiol Regul Integr Comp Physiol 297(3): R587-R592.

4. Kreijkamp-Kaspers S, Kok L, Grobbee DE, de Haan EH, Aleman A Lampe JW, van der Schouw YT (2004) Effect of Soy Protein Containing Isoflavones on Cognitive Function, Bone Mineral Density, and Plasma Lipids in Postmenopausal Women. A Randomized Controlled Trial. JAMA 292(1):65-74.

5. Danilovich N, Sairam MR (2005) Recent female mouse models displaying advanced reproductive aging. Exp Gerontol 41(2): 117-122.

6. Renaud TF, Benefice FL, De Meeü ST, Gueganl JF (2007) Internationa Variability of Ages Menarche and Menopause: Patterns and Main Determinants. Human Biology 73(2): 271-290.

7. Wang H, Murphy PA (1994) Isoflavone Content in Commercial Soybean Foods. Journal of. Agricultural Food and Chemistry 42(8): 1666-1673.

8. Sunita P, Pattanayak SP (2011) Phytoestrogens in postmenopausal indications: A theoretical perspective. Pharmacognosy Rev 5(9): 4147.
9. Kurzer MS (2000) Hormonal Effects of Soy Isoflavones: Studies in Premenopausal and postmenopausal Women. J Nutr 130(3): 660S-661S.

10. Johnson K, Vemuri S, Alsahfi S, Castillo R, Cheriyath V(2016) Glyconerich Soy Isoflavone Extracts Promote Estrogen Receptor Positive Breast Cancer Cell Growth. Nutr Cancer 68(4):622-633.

11. Van Kempena TA, Milnera TA, Waters EM (2011) Accelerated Ovarian Failure: A novel, chemically induced animal model of menopause. Brain Res 1379: 176-187.

12. Lohff JC, Christian PJ, Marion SL, Arrandale A, Hoyer PB (2005) Characterization of Cyclicity and Hormonal Profile with Impending Ovarian Failure in a Novel Chemical- Induced Mouse Model of Perimenopause. Comp Med 55(6): 523-527.

13. Hoyer PB, Devine PJ, Hu X, Thompson KE, Sipes GI (2001) Ovarian Toxicity of 4-Vinylcyclohexene Diepoxide: A Mechanistic Model. Toxicol Pathol 29(1): 91-99.

14. Hoyer PB, Mayer LP (2011) Mouse model for perimenopause and menopause and methods of preparation. Google Patents.

15. McLean AC, Valenzuela N, Fai S, Bennett SA (2012) Performing Vaginal Lavage, Crystal Violet Staining, and Vaginal Cytological Evaluation for Mouse Estrous Cycle Staging Identification. J Vis Exp (67): e4389.

16. Gosden RG, Laing SC, Felicio LS, Nelson JF, Finch CE (1983) Imminen Oocyte Exhaustion and Reduced Follicular Recruitment Mark the Transition to Acyclicity in Aging C57BL/6J Mice. Biol Reprod 28(2): 255-260.

\section{Your next submission with Juniper Publishers will reach you the below assets}

- Quality Editorial service

- Swift Peer Review

- Reprints availability

- E-prints Service

- Manuscript Podcast for convenient understanding

- Global attainment for your research

- Manuscript accessibility in different formats ( Pdf, E-pub, Full Text, Audio)

- Unceasing customer service

Track the below URL for one-step submission https://juniperpublishers.com/online-submission.php 